\title{
A Phase II Study of Antineoplastons A10 and AS2-1 in Adult Patients with Primary Brain Tumors-Final Report (Protocol BT-09)
}

\author{
Stanislaw R. Burzynski, Tomasz J. Janicki, Gregory S. Burzynski \\ Burzynski Clinic, Houston, Texas, USA \\ Email: srb@burzynskiclinic.com
}

Received 21 October 2015; accepted 22 November 2015; published 25 November 2015

Copyright (C) 2015 by authors and Scientific Research Publishing Inc.

This work is licensed under the Creative Commons Attribution International License (CC BY). http://creativecommons.org/licenses/by/4.0/

c) (i) Open Access

\section{Abstract}

Antineoplastons A10 and AS2-1 (ANP) are synthetic derivatives of glutamine, isoglutamine, and phenylacetic acid. In 1993, a phase II clinical trial program began according to protocols based on the initial protocol, BT-06, which was transferred from the National Institutes of Health (NIH). Protocol BT-09 was designed for different types of primary brain tumors in adults that were not curable by standard treatment. The study was designed as a single arm, two-stage, phase II trial of ANP as a monotherapy in a high-risk, poor-prognosis population. The total number of registered subjects was 40 . The majority of patients were diagnosed with high-grade tumors $(N=33)$. In this group, 12 patients carried diagnosis of anaplastic astrocytoma (AA) and 11 patients of glioblastoma. In the group of low-grade tumors $(N=7)$, there were 6 cases of low-grade glioma, and 1 neurocytoma grade 2 . A group of 12 patients did not receive any prior treatment, 12 patients had surgical resection only, 5 patients received radiation therapy (RT) only, and 11 patients received both RT and chemotherapy. The median duration of ANP was 16.6 weeks. The median dosage of A10 was $7.16 \mathrm{~g} / \mathrm{kg} / \mathrm{d}$ and $\mathrm{AS2}-1$ was $0.27 \mathrm{~g} / \mathrm{kg} / \mathrm{d}$. Responses were accessed by gadolinium-enhanced magnetic resonance imaging (MRI). Objective responses (OR) in all patients were $22.5 \%$ and in the AA group were $41.7 \%$ of patients. The median progression-free survival (PFS) in the AA group was 5.4 months. The median overall survival (OS) was 12.7 months and $O S$ at 1 and 2 years was $54.5 \%$ and $45.5 \%$ correspondingly. The treatment was well-tolerated with reversible grade 3 and 4 toxicities in $35 \%$ of all patients $(N=40)$. In conclusion, the study reached efficacy endpoint and ANP was well-tolerated and compared favorably to the current treatment of AA.

\section{Keywords}

Anaplastic Astrocytoma, Antineoplastons A10 and AS2-1, Low-Grade Astrocytoma, Phase II Clinical Trial, Tectal Glioma 


\section{Introduction}

Antineoplastons A10 and AS2-1 (ANP) are synthetic derivatives of glutamine, isoglutamine and phenylacetic acid [1]. A10 injections consist of a mixture of the sodium salts of phenylacetylglutamine (PG) and phenylacetylisoglutamine (isoPG) in a 4:1 ratio. AS2-1 is a mixture of phenylacetate sodium (PN) and PG in a 4:1 ratio. ANPs are multi-targeted medications affecting the hubs of the gene expression network, multiple signaling pathways, the cell cycle, cell death, and cellular and nuclear transports, thereby resulting in a better chance for durable cancer control. For over 30 years, our team was developing multi-targeted therapies with antineoplastons including basic research, Phase I and Phase II studies [1]. Phase II studies of ANP injections in astrocytoma began in 1988 and documented objective responses (OR) in 30\% of 20 patients diagnosed with anaplastic astrocytoma (AA), low-grade astrocytoma (LGA), and diffuse intrinsic pontine glioma (DIPG) [2]. In 1993, a Phase II clinical trials program began according to protocols based on initial Protocol BT-06 which was transferred from the National Institutes of Health (NIH) to Burzynski Research Institute, Inc. (BRI) [3]. Among the initial protocols, BT-09 and BT-10 were designed for different types of primary brain tumors which were not curable by standard treatment in adults (BT-09) and children (BT-10). Numerous additional protocols dealt with the treatment of specific types of brain tumors in children and adults and colorectal cancer [4]-[18]. The aim of this single arm study is to assess the efficacy and safety of ANP in adults who have different types of incurable primary brain tumors. Special emphasis was directed to high-grade tumors (82.5\% of patients) with attention to the subgroup of AA.

Approximately 5600 people in the United States were diagnosed with AA in years 2007-2011 [19]. AA is classified as a World Health Organization grade 3 neuroepithelial tumor [20]. Initial standard therapy for AA includes surgical resection followed by radiation therapy (RT) and chemotherapy [21]. Nitrosourea treatment provided the first successful therapy for AA, but since 1999 temozolomide (TMZ) became the most frequently used therapeutic agent due to better toxicity profile and comparable efficacy [21] [22]. Unfortunately, the remissions from treatment with TMZ are temporary with progression-free survival (PFS) of $24 \%$ in twelve months [21]. Ultimately, almost all patients will relapse and die from progressive tumor. Recurrent AA remains an incurable brain tumor. The use of TMZ has been criticized recently, and there seems to be a return to the 20-year-old nitrosourea-based chemotherapy [23] [24]. In approximately 30\% of patients, chemotherapy may cause irreversible toxicity which may seriously compromise the quality of life [25] [26]. New treatment options for AA are desperately needed.

\section{Patients and Method}

\subsection{Patient Population}

The study accepted patients with brain tumors for whom curative therapeutic regimens did not exist. Patients who had been previously treated with other therapies, as well as not previously treated were eligible.

Recruited patients were over 18 years of age with radiologic evidence of residual, recurrent or progressive tumor by gadolinium-enhanced magnetic resonance imaging (MRI) performed within 14 days before initiating the study, and no earlier than four weeks following tumor resection. At least eight weeks must have elapsed since the last dose of RT and at least four weeks since the last dose of chemotherapy (six weeks for nitrosoureas) or immunotherapy. There should be a complete recovery and at least four weeks from prior surgical procedure.

The patients should have histological confirmation of brain tumors; the only exceptions are some cases of dangerous location of the tumor. Confirmation of the pathologic diagnosis by an outside pathologist was also required. WHO criteria for high-grade and low-grade tumors were used.

The additional inclusion, exclusion and removal from the study criteria were the same as in the other brain tumor study [11]. All study subjects and/or guardians read, understood, and signed written informed consent prior to enrollment. This study was conducted in accordance with the U.S Code of Federal Regulations, Title 21, Parts 50, 56, and 312; the Declaration of Helsinki (1964) including all amendments and revisions; the Good Clinical Practices: Consolidated Guideline (E6); International Conference on Harmonization; and the FDA's Guidance for Industry. The study was sponsored by BRI and conducted by the Burzynski Clinic (BC) in Houston, Texas. The patients were enrolled based on their physician's referral or were self-referred and they did not pay for the investigational agents. 


\subsection{Study Design}

The study was designed as a single-arm, two-stage, interventional Phase II trial of ANP as the monotherapy in a high-risk, poor-prognosis population [27]. The study was listed by the National Cancer Institute (NCI). It was supervised by the independent Institutional Review Board (BRI-IRB, BC-BT-09).

The study was performed according to Protocol BT-09 which was submitted to the FDA under the IND 43,742. The study started on July 23, 1996, and ended accrual on March 14, 2011. Subsequently, the protocol was amended by BRI several times; however, none of the amendments altered the aim or design of the original study objectives/outcomes.

\subsection{Statistical Considerations}

The sample size was calculated based upon the method described by Chang et al. [27]. A response rate to ANP of $\geq 10 \%$ was considered "of interest" and the primary endpoint was to determine the objective response rate (confirmed complete response (CR) or partial response (PR)) to ANP therapy. An interim analysis would be conducted after 20 subjects had enrolled in the study. If 1 or more patients achieved a confirmed radiographic response, an additional 20 subjects would be recruited. Survival was measured from the first day of ANP administration until death from any cause, and time to treatment failure was likewise measured from the first day of the treatment until the date of first observation of progressive disease (PD) or death from any cause whichever came first. The distributions of survival and treatment failure were estimated by Kaplan-Meier analysis.

\subsection{Treatment Plan}

ANP (A10 and AS2-1) were the only anti-cancer treatments administered in this study. The formulations were delivered via a dual-channel infusion pump and single-lumen subclavian catheter (Broviac or Groshong) every 4 hours. The patients were advised to continue the treatment until the maximum response was achieved and for eight months thereafter. The details of administration of ANP were published before [11].

Medications that were considered necessary for the subjects' welfare and that did not interfere with the evaluation of treatment were given at the discretion of the investigator. The use of corticosteroids was carefully monitored. Treatment with other antineoplastic or immunomodulatory agents was not permitted during the study. Subjects received full supportive care, including transfusions of blood products and antibiotics when appropriate. No other anticancer mediation was permitted.

\subsection{Evaluation and Follow-Up}

Prior to the start of treatment, a gadolinium-enhanced MRI measured all contrast-enhancing lesions. The products of the two greatest perpendicular diameters of all lesions were calculated and totaled, providing a baseline evaluation for each study subject. As a common practice at that time in other clinical trials, the tumor measurements were based on contrast enhanced lesions, but the overall tumor size was also measured including T2 and FLAIR images [28] [29]. In cases of the non-enhanced signal, the two greatest perpendicular diameters of T2 weighted lesions were calculated and totaled, providing a baseline evaluation for each study subject as described above. A positron emission tomography (PET) scan was used also to evaluate the overall response in such cases. The baseline provided the reference for determining response outcomes to the treatment. The details of laboratory tests and additional examinations were published before [11]. Toxicity was evaluated in all patients enrolled in the study (intent-to-treat (ITT) population).

In accordance with other Phase II studies conducted at the initiation of this trial, the possible responses to the treatment were CR, PR, stable disease (SD) and PD. CR required the disappearance of all enhancing lesions, sustained for at least 4 weeks, and only physiologic replacement doses of steroids were acceptable. PR required $50 \%$ or higher decrease of the sum of the products of the two largest perpendicular diameters of enhancing lesions and stable or reduced corticosteroid doses. PD was determined when there was over $50 \%$ increase of enhancing lesions or new lesions, and SD was the status between PR and PD. The results of all MRI and PET scans were verified by radiologists not affiliated with BRI or BC and their determination of response was accepted including one case of nonenhanced tumor. Study subjects were categorized by their overall best response during the course of the treatment. The duration of each response was measured from the date that the criteria 
for the outcome were first met until the date that PD was first documented. The original protocol required more than $50 \%$ increase of tumor size for PD, but in this publication $25 \%$ increase was acceptable and all cases reported in this paper were re-evaluated by RANO criteria to allow comparison with other studies. In the case of $\mathrm{SD}$, the duration was measured from the time therapy commenced.

During the study the generally accepted criteria for evaluation of responses in high-grade glioma (HGG) changed toward reliance on overall survival (OS) and PFS rather than tumor responses. As a result, this report includes also survival analysis.

Correlative studies including 1p19q deletions and MGMT silencing were not performed because they were not yet introduced when the patients were accrued except for the patients who were accrued before completion of the study.

The primary endpoint was disease response, and all study subjects were evaluable for toxicity. Study subjects who received $\geq 12$ weeks of therapy or developed an objective response (CR or PR) were further analyzed. Time to best response, dosage, and duration of best response were the variables of interest.

The average maximum effective daily dose, the range, standard deviation for that average, and the $95 \% \mathrm{CI}$ were calculated.

For OS analysis, a Kaplan-Meier curve with 95\% CI was created using MedCalc Statistical Software version 14.12.0 [30].

\section{Results}

\subsection{Patient Demographics}

Patient demographics are shown in Table 1. As of March 9, 2012, all subjects were removed from the therapy due to a CR, SD, PD, subject request, worsening clinical condition, or death.

The 40 eligible subjects (22 men and 18 women) who met enrollment criteria (Table 1) had a median age of 36 years. 38 subjects were of Caucasian ethnicity, 1 African American and 1 Latin American. Six subjects presented with multifocal disease and leptomeningeal involvement and 1 had multicentric disease.

The majority of patients were diagnosed with high-grade tumors $(N=33)$. Among them, the largest group was anaplastic glioma (AG) which included 14 patients. In this group 12 patients (86\%) were diagnosed with AA. GBM was represented by 11 patients and the additional high-grade tumors included primitive neuroectodermal tumors (PNET) $N=3$, DIPG $N=3$, and tectal glioma (TG) $N=1$.

In the group of low-grade tumors, there were six cases of low-grade glioma (LGG).

12 patients did not receive prior treatment and 12 had surgical resection only. 5 patients received RT as a single treatment and 11 patients were treated with RT and chemotherapy. 8 patients received a single chemotherapy regimen, but 3 patients failed 2, 4 and 5 lines of chemotherapy. 1 of these patients failed high dose chemotherapy and stem cell rescue and another was treated with targeted therapy. The total of 7 patients failed TMZ.

\subsection{Treatment}

The maximum tolerated daily dosage of ANP A10 $(N=40)$ ranged from $3.92-18.04 \mathrm{~g} / \mathrm{kg} / \mathrm{d}$, with a median of $9.91 \mathrm{~g} / \mathrm{kg} / \mathrm{d}$. For AS2-1, the median maximum tolerated daily dose was $0.34 \mathrm{~g} / \mathrm{kg} / \mathrm{d}$, with a range of $0.17-0.45$ $\mathrm{g} / \mathrm{kg} / \mathrm{d}$.

The median daily dose of A10 to obtain OR $(N=9)$ was $7.04 \mathrm{~g} / \mathrm{kg} / \mathrm{d}$ (range $4.74-12.48 \mathrm{~g} / \mathrm{kg} / \mathrm{d}$ ), standard deviation $=2.55$, and $95 \%$ confidence interval $(\mathrm{CI})=1.67$.

The median daily dose of AS2-1 to obtain OR $(N=9)$ was $0.25 \mathrm{~g} / \mathrm{kg} / \mathrm{d}$ (range $0.22-0.36 \mathrm{~g} / \mathrm{kg} / \mathrm{d}$ ), standard deviation $=0.05$, and $95 \%$ confidence interval $(\mathrm{CI})=0.03$.

One patient was evaluated as CR, 8 weeks after discontinuation of ANP IV and while on an oral formulation of ANP, but the beginning of PR was observed 4 months after the start of ANP IV.

For subjects with SD $(N=12)$ the median daily dose of A10 was $12.04 \mathrm{~g} / \mathrm{kg} / \mathrm{d}$ (range $2.2-12.04 \mathrm{~g} / \mathrm{kg} / \mathrm{d}$, standard deviation $=3.19,95 \% \mathrm{CI}=1.80$ ); the median daily dose of AS2-1 was $0.24 \mathrm{~g} / \mathrm{kg} / \mathrm{d}$ (range $0.16-0.41$ $\mathrm{g} / \mathrm{kg} / \mathrm{d}$, standard deviation $=0.08,95 \% \mathrm{CI}=0.05$ ).

The higher median values of A10 and AS2-1 for the entire population $(9.91 \mathrm{~g} / \mathrm{kg} / \mathrm{d} ; 0.34 \mathrm{~g} / \mathrm{kg} / \mathrm{d}$, respectively) compared to median doses of A10 and AS2-1 recorded until the best responses $(7.04 \mathrm{~g} / \mathrm{kg} / \mathrm{d} ; 0.25 \mathrm{~g} / \mathrm{kg} / \mathrm{d})$ can be 
Table 1. Patient demographics and prior treatment in ITT.

\begin{tabular}{|c|c|c|c|c|c|c|}
\hline & \multicolumn{4}{|c|}{ ENROLLED SUBJECTS $(N=40)$} & & \\
\hline & \multicolumn{2}{|c|}{ All patients } & \multicolumn{2}{|c|}{ High-Grade Tumors $(N=33)$} & \multicolumn{2}{|c|}{ Low-Grade Tumors $(N=7)$} \\
\hline & \multicolumn{6}{|c|}{ Demographic Characteristic } \\
\hline \multicolumn{7}{|l|}{ Age (years) } \\
\hline Median & \multicolumn{2}{|c|}{36.0} & \multicolumn{2}{|c|}{36.7} & \multicolumn{2}{|c|}{35.2} \\
\hline Range & \multicolumn{2}{|c|}{$18.0-64.1$} & \multicolumn{2}{|c|}{$18.0-60.8$} & \multicolumn{2}{|c|}{$22.1-64.1$} \\
\hline \multicolumn{7}{|l|}{ Sex } \\
\hline Male & \multicolumn{2}{|c|}{22} & \multicolumn{2}{|c|}{20} & \multicolumn{2}{|c|}{2} \\
\hline Female & \multicolumn{2}{|c|}{18} & \multicolumn{2}{|c|}{13} & \multicolumn{2}{|c|}{5} \\
\hline \multirow[t]{3}{*}{ Ethnicity } & \multicolumn{2}{|c|}{ W-38, B-1, M-1 } & \multicolumn{2}{|c|}{ W-31, B-1, M-1 } & \multicolumn{2}{|c|}{$\mathrm{W}-7$} \\
\hline & \multicolumn{6}{|c|}{ Karnofsky Performance Score } \\
\hline & $\mathrm{BT}$ & $\mathrm{AT}$ & $\mathrm{BT}$ & AT & $\mathrm{BT}$ & AT \\
\hline Median & 70 & 80 & 80 & 95 & 70 & 70 \\
\hline \multirow[t]{2}{*}{ Range } & $60-100$ & $40-100$ & $60-100$ & $80-100$ & $60-90$ & $40-100$ \\
\hline & \multicolumn{6}{|c|}{ Prior Treatment } \\
\hline None & \multicolumn{2}{|c|}{12} & \multicolumn{2}{|c|}{9} & \multicolumn{2}{|c|}{3} \\
\hline Surgery only & \multicolumn{2}{|c|}{12} & \multicolumn{2}{|c|}{11} & \multicolumn{2}{|c|}{1} \\
\hline Surgery and RT and CH & & & & & & \\
\hline RT only & & & & & & \\
\hline Surgery and RT & & & & & & \\
\hline $\mathrm{RT}$ and $\mathrm{CH}$ & & & & & & \\
\hline & & & Chemothe & racteristics & & \\
\hline 1 line & & & & & & \\
\hline 2 lines & & & & & & \\
\hline 4 and 5 lines & & & & & & \\
\hline & & & Addit & atment & & \\
\hline HDC and SCR & & & & & & \\
\hline
\end{tabular}

Note: AT—at completion, BT—at baseline, B-African-American, CH—chemotherapy, HDC—high-dose chemotherapy, M-Latin-American, RT—radiation therapy, SCR—stem cell rescue, $\mathrm{W}$-Caucasian.

explained by design of the protocol which required to increase the daily dose of ANP until the maximum tolerated dose (not exceeding $20 \mathrm{~g} / \mathrm{kg} / \mathrm{d}$ ).

\subsection{Response and Survival}

Out of 40 subjects, there were 4 CR (10\%), 5 PR (12.5\%), 12 SD (30\%), 10 PD (25\%), and 9 cases (22.5\%) were not further analyzed; they were withdrawn from the study, and did not have follow-up evaluations including MRI. The overall objective response rate (OR) was $22.5 \%$, and the overall disease control rate which includes CR, PR and SD was 52.5\%.

In the group of high-grade tumors of 33 patients, there were 3 CR (9.1\%), 5 PR (15.2\%), 8 SD (24.2\%), 10 PD (30.3\%), and 7 (21.2\%) nonevaluable cases. The OR rate was $24.3 \%$, and the disease control rate was $48.5 \%$. 
In the low-grade tumor group of 7 patients, there was 1 CR (14.3\%), no PR, 4 SD (57.1\%), no PD, and 2 nonevaluable cases (28.6\%). The disease control rate $71.4 \%$.

The response rates are presented in Table 2(a) and Table 2(b).

The most important results were noticed in the subgroup of 12 patients with AA (grade 3). There were 2 CRs (16.7\%), 3 PRs (25\%), 3 SDs (25\%), 3 PDs (25\%), and 1 (8.1\%) nonevaluable case. The OR rate was 41.7\%, and the disease control rate $66.7 \%$. The OR group was represented by 4 newly diagnosed and one recurrent AA. Most of these patients ( 9 cases) were newly-diagnosed, and 5 patients had biopsy only. One of the patients who received prior RT responded with over $90 \%$ decrease of the products compared to baseline for a period of one and half year (between March 1997 and August 1998) with one MRI showing complete disappearance of the lesions (Figure 1).

Subsequently this patient progressed and died 18 months after discontinuation of ANP due to tumor recurrence and transformation to GBM. The second patient treated by RT did not respond to the treatment and the third patient treated previously with combination of RT and chemotherapy responded to the treatment with initial stabilization followed by progression.

In other high grade tumor group of 11 patients with GBM, there were no OR, 1 SD (9.1\%), 6 PD (54.5\%) and 4 (36.4\%) nonevaluable cases.

In the remaining group of 9 patients with other types of high-grade tumors, 2 groups were observed: DIPG (3 patients) and PNET with 3 patients with recurrent medulloblastoma. There was 1 SD in newly-diagnosed DIPG and 1 PR and 1 SD in the PNET sub group.

Due to the low number of patients these groups were not analyzed any further.

The remaining 2 patients were diagnosed with high-grade malignant glioma (the biopsy was not done due to high risk of hemorrhage, and one interesting case of TG, previously not treated, who obtained CR and was described separately [16].

OS and PFS were analyzed for ITT and selected groups. OS was calculated from start of ANP until death from any cause, PFS was calculated from start of ANP till first radiologically documented progression, start of other treatment or death (Figure 2 and Figure 3).

The median OS in a group of 40 patients was 11.9 months (95\% CI 8.6 - 32.7) and OS at 6 months, 1, 2, 5, and over 10 years was $70 \%, 50 \%, 40 \%, 22.5 \%$ and $14.6 \%$ correspondingly.

The median OS in the group of AA (12 patients) was 25.4 months (95\% CI 9.7 - 45.1) and OS at 6 months, 1, and 2 years was $83.3 \%, 58.3 \%, 50.0 \%$ correspondingly.

Table 2. (a) Response rates; (b) Response rates in the anaplastic glioma group of high-grade tumors.

(a)

\begin{tabular}{|c|c|c|c|c|c|c|}
\hline \multirow[b]{2}{*}{ Group } & \multicolumn{6}{|c|}{ Response $N(\%)$} \\
\hline & $N$ & CR & PR & SD & PD & $\mathrm{NE}$ \\
\hline High-Grade Tumors & 33 & $3(9.1)$ & $5(15.2)$ & $8(24.2)$ & $10(30.3)$ & $7(21.2)$ \\
\hline Low-Grade Tumors & 7 & $1(14.3)$ & 0 & $4(57.1)$ & 0 & $2(28.6)$ \\
\hline Total & 40 & $4(10)$ & $5(12.5)$ & $12(30)$ & $10(25)$ & $9(22.5)$ \\
\hline
\end{tabular}

Note: CR—complete response, NE—nonevaluable, $\mathrm{PR}$ — partial response, $\mathrm{PD}$ —progressive disease, SD—stable disease.

(b)

\begin{tabular}{ccccccc}
\hline & \multicolumn{7}{c}{ Response $N(\%)$} \\
Group & $N$ & CR & PR & SD & PD & NE \\
\hline AA & 12 & $2(16.7)$ & $3(25.0)$ & $3(25.0)$ & $3(25.0)$ & $1(8.3)$ \\
AMG & 2 & & & $1(50.0)$ & & $1(50.0)$ \\
Total (AG) & 14 & $2(14.3)$ & $3(21.4)$ & $4(28.6)$ & $3(21.4)$ & $2(14.3)$ \\
\hline
\end{tabular}

Note: AA—anaplastic astrocytoma, AMG—anaplastic mixed glioma, CR—complete response, NE—nonevaluable, PR—partial response, PD—progressive disease, $\mathrm{SD}$ - stable disease. 


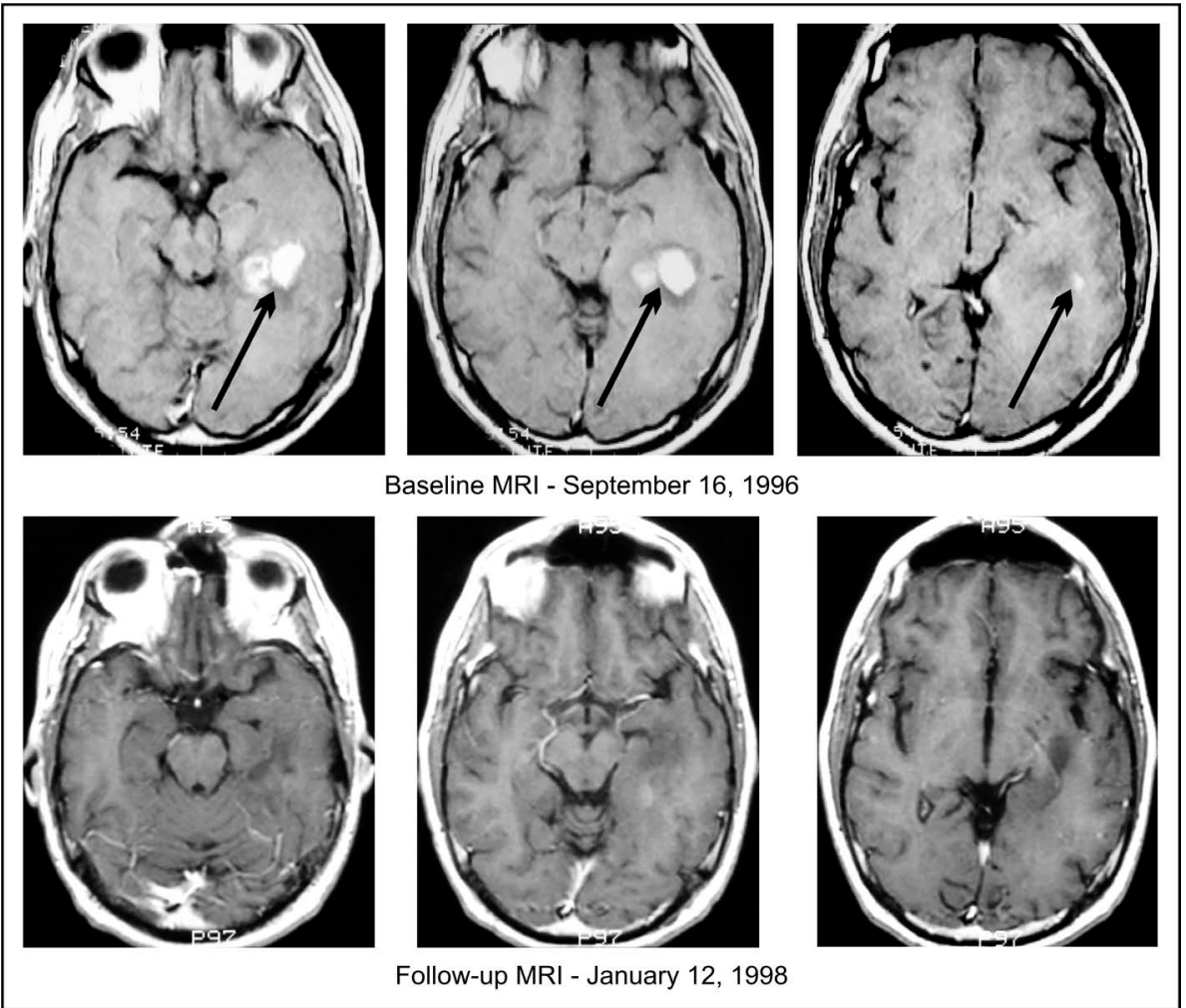

Figure 1. Anaplastic astrocytoma in a 26-year-old male (Case 5) who progressed after RT. Post-contrast MRIs of the head. The follow-up MRI documents a complete resolution of the tumor. Arrows indicate the tumor.

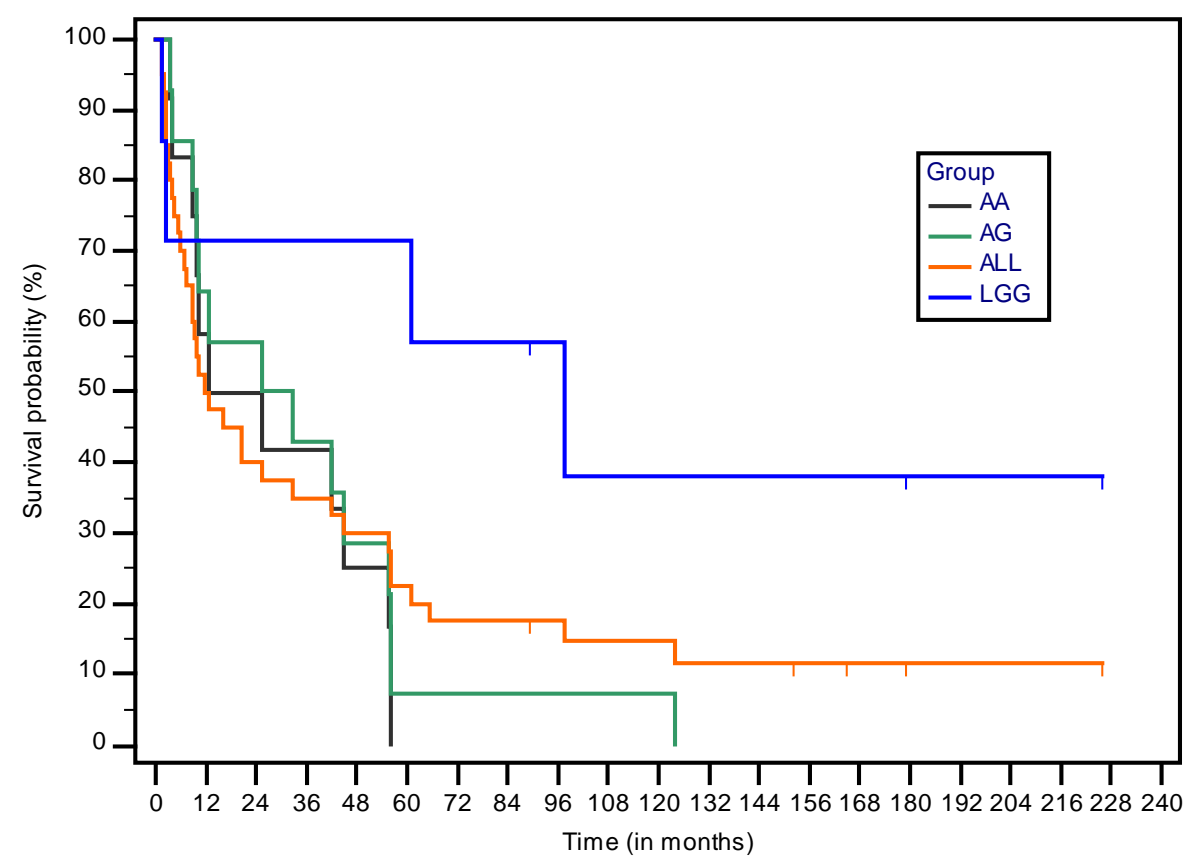

Figure 2. Overall survival in ITT and selected groups.

The median OS in group of AG (14 patients) was 25.3 months (95\% CI 9.7 - 55.5) and OS at 6 months, 1, 2, 5, and over 10 years was $85.7 \%, 64.35 \%, 57.1 \% 7.1 \%$ and $7.1 \%$ correspondingly. 


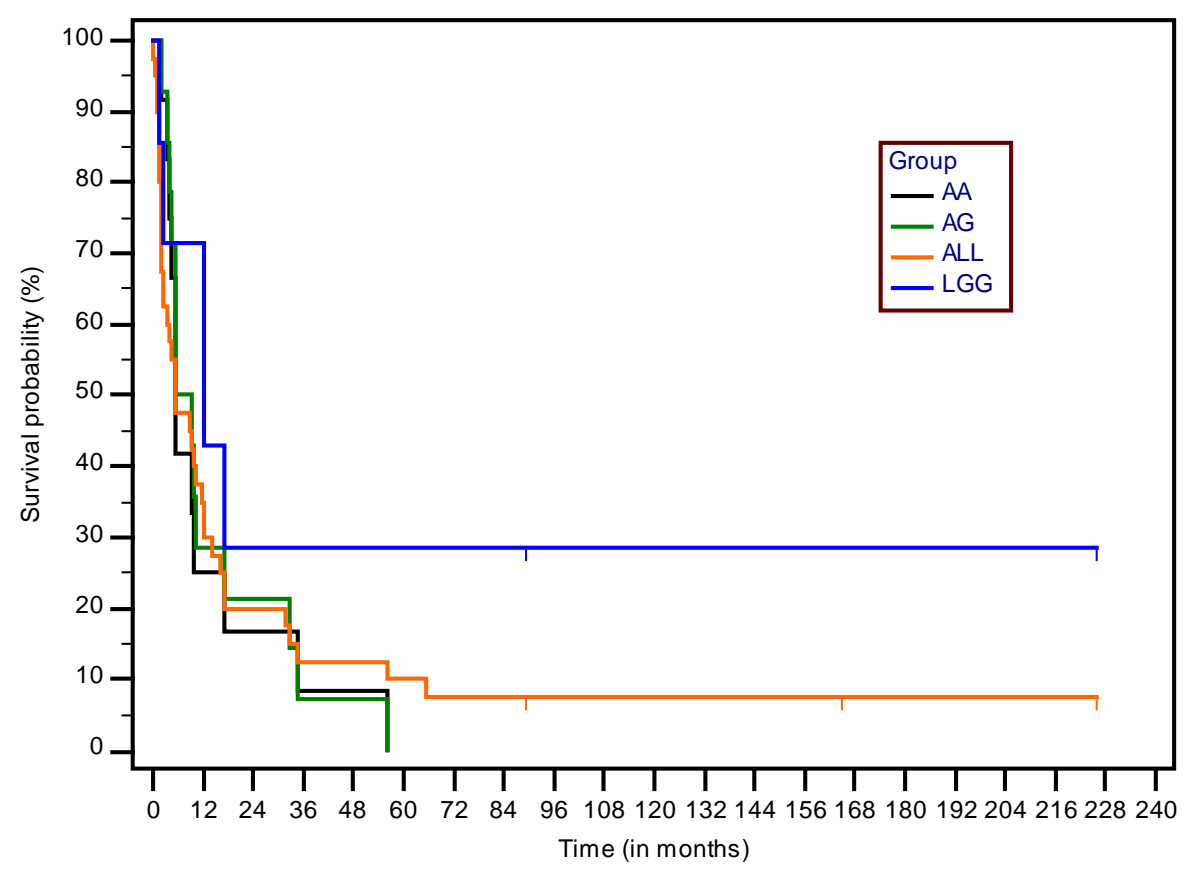

Figure 3. Progression-free survival in ITT and selected group.

The median OS in group of GBM (11 patients) was 5.2 months (95\% CI 2.2 - 7.1) and OS at 6 months and 1 year was $36.4 \%$ and $9.1 \%$ correspondingly.

The median OS in group of LGG (7 patients) was 97.3 months (95\% CI $2.4-97.3$ ) and OS at 6 months, 1, 2, 5 , and over 10 years was $71.4 \%, 71.4 \%, 71.4 \%, 71.4 \%$ and $38.1 \%$ correspondingly.

The median PFS in a group of 40 patients was 5.4 months (95\% CI $2.4-11.9$ ), at 6 months $47.5 \%$, 1 year 35\% and at 2 years $20 \%$.

The median PFS in the group of AA (12 patients) was 5.4 months (95\% CI 4.3 - 9.7) , 6 months $41.7 \%$, at 1 year $25.0 \%$ and at 2 years $16.7 \%$.

The median PFS in the group of AG (14 patients) was 5.5 months (95\% CI 4.3 - 16.8), 6 months 50.0\%, at 1 year $28.6 \%$ and at 2 years $21.4 \%$.

The median PFS in the group of GBM (11 patients) was 1.6 months (95\% CI 1.0 - 2.1) and at 6 months it was $9.1 \%$.

The median PFS in the group of LGG (7 patients) was 12.2 months (95\% CI 2.4 - 17.2), 6 months $71.4 \%$, at 1 year $71.4 \%$ and at 2 years $28.6 \%$.

\subsection{Adverse Events}

Safety assessments were analyzed based upon the total number of enrolled patients in the study $(N=40)$ (Table 3). All adverse drug events (ADEs) have been coded according to Common Terminology Criteria for Adverse Events v3 (CTCAEv3).

The treatment was well tolerated. There were grade 3 toxicities consisting of hypernatremia, hypokalemia fatigue, arthralgia, somnolence, urinary frequency and urticaria in 35\% patients and grade 4 toxicities consisting of hypernatremia, hypocalcemia and hypokalemia in $12.5 \%$ patients. ADEs were completely reversible.

There were no chronic toxicities.

\section{Discussion}

The largest group of patients treated under the BT-09 Protocol consisted of 14 cases of AG (the majority, 12 had AA). Clinical trials and meta-analyses confirmed the importance of chemotherapy in the treatment of patients with malignant glioma [33]-[35]. However, beneficial effect in patients with AA is not supported by strong evidence [36]. During the last eight years, the results of pharmacological treatments without RT for newly-diag- 
Table 3. Incidence of adverse drug experiences, grades 3, 4, or 5, reported by patients in the BT-09 study compared to other studies of chemotherapy for anaplastic glioma.

\begin{tabular}{|c|c|c|c|c|c|c|c|}
\hline \multirow[t]{2}{*}{$\mathrm{ADE}$} & \multicolumn{2}{|c|}{$\begin{array}{c}\text { Burzynski et al. } \\
2015 \text { Antineoplastons } \\
\text { A10 and AS2-1 } \\
N(\%)\end{array}$} & \multicolumn{2}{|c|}{$\begin{array}{c}\text { Chamberlain et al. [31] } \\
2006 \\
\text { Cyclophosphamide } \\
N(\%)\end{array}$} & \multicolumn{2}{|c|}{$\begin{array}{c}\text { Chamberlain et al. [32] } \\
2008 \\
\text { CPT-11 } \\
N(\%)\end{array}$} & \multirow{2}{*}{$\begin{array}{c}\text { Yung et al. [22] } \\
1999 \\
\text { Temozolomide } \\
N(\%) \\
\text { Grades } 3 / 4\end{array}$} \\
\hline & Grade 3 & Grade 4 & Grade 3 & Grade 4 & Grades 3/4 & Grade 5 & \\
\hline Hypernatremia & $5(12.5)$ & $2(5)$ & & & & & \\
\hline Hypocalcemia & & $1(2.5)$ & & & & & \\
\hline Hypokalemia & $4(10)$ & $2(5)$ & & & & & \\
\hline Thrombocytopenia & & & $5(12)$ & $1(2)$ & $2(5)$ & & $10(6)$ \\
\hline Neutropenia & & & $7(17)$ & $1(2)$ & $1(2)$ & & $3(2)$ \\
\hline Leukopenia & & & $15(37)$ & $2(5)$ & $1(2)$ & & $3(2)$ \\
\hline Anemia & & & $5(12)$ & & & & $1(1)$ \\
\hline Asthenia & & & & & $2(5)$ & & $9(6)$ \\
\hline Fatigue & $1(2.5)$ & & $9(22)$ & & & & $7(5)$ \\
\hline Fever & & & & & & & $3(2)$ \\
\hline Peripheral edema & & & & & & & $1(1)$ \\
\hline \multicolumn{8}{|l|}{ Weight loss } \\
\hline Headache & & & $1(2)$ & & & & $10(6)$ \\
\hline Convulsions & & & $1(2)$ & & & & $8(5)$ \\
\hline Dizziness & & & & & & & $1(1)$ \\
\hline Somnolence & $1(2.5)$ & & & & & & $5(3)$ \\
\hline \multicolumn{8}{|l|}{ Suicide attempt } \\
\hline Pain & $1(2.5)$ & & & & & & \\
\hline Other neurological $\mathrm{ADE}^{*}$ & & & & & & & $2(1)$ \\
\hline \multicolumn{8}{|l|}{ Hypertension } \\
\hline \multicolumn{8}{|l|}{ Syncope } \\
\hline \multicolumn{8}{|l|}{ Bradycardia } \\
\hline \multicolumn{8}{|l|}{ Apnea } \\
\hline Anorexia & & & & & & & $1(1)$ \\
\hline Constipation & & & $1(2)$ & & & & $1(1)$ \\
\hline Diarrhea & & & & & $3(7)$ & $1(2)$ & $3(2)$ \\
\hline Nausea & & & $3(7)$ & & $2(5)$ & & $16(10)$ \\
\hline Vomiting & & & $2(5)$ & & $4(10)$ & & $10(6)$ \\
\hline Pruritus & & & & & & & $2(1)$ \\
\hline Infection & & & $1(2)$ & & $1(2)$ & & \\
\hline Thrombophlebitis & & & $1(2)$ & & $1(2)$ & & \\
\hline \multicolumn{8}{|l|}{ Intracranial hemorrhage } \\
\hline \multicolumn{8}{|l|}{ Bilirubinemia } \\
\hline \multicolumn{8}{|l|}{ Hyperglycemia } \\
\hline Urinary frequency & $1(2.5)$ & & & & & & \\
\hline Urticaria & $1(2.5)$ & & & & & & \\
\hline
\end{tabular}

Note: *Other neurological adverse drug events (ADEs): ataxia, impaired cognition, speech disorder, behavior disorder, altered mental status. 
Table 4. Response to antineoplaston treatment compared to phase II studies with chemotherapy for anaplastic glioma.

\begin{tabular}{|c|c|c|c|c|c|c|c|c|c|}
\hline \multirow[b]{2}{*}{ Author/Treatment } & \multicolumn{4}{|c|}{ Efficacy Response (\%) } & \multicolumn{5}{|c|}{ Survival (Probability \%) } \\
\hline & OR & SD & $\begin{array}{l}\text { PFS } \\
\text { Median }\end{array}$ & $\begin{array}{c}\text { OS } \\
\text { Median }\end{array}$ & $\begin{array}{c}\text { PFS } \\
6 \text { months }\end{array}$ & $\begin{array}{c}\text { OS } \\
6 \text { months }\end{array}$ & $\begin{array}{c}\text { PFS } \\
12 \text { months }\end{array}$ & $\begin{array}{c}\text { OS } \\
12 \text { months }\end{array}$ & 2 years \\
\hline $\begin{array}{c}\text { Chamberlain et al. } 2006 \text { [31] } \\
\text { Cyclophosphamide }\end{array}$ & 23 & 40 & 4 & 8 & 30 & - & 8 & 8 & \\
\hline $\begin{array}{l}\text { Chamberlain et al. } 2008 \text { [32] } \\
\text { CPT-11 }\end{array}$ & 13 & 85 & 4.1 & 6.9 & 40 & - & 5 & 23 & \\
\hline $\begin{array}{l}\text { Yung et al. } 1999 \text { [22] } \\
\text { Temozolomide after first relapse }\end{array}$ & 33 & 31 & 5.5 & 14.2 & 49 & 78 & 24 & 56 & \\
\hline $\begin{array}{l}\text { Burzynski et al. } 2015 \\
\text { Antineoplastons A10 and AS2-1 } \\
\text { Anaplastic Astrocytoma Group }\end{array}$ & 41 & 25 & 5.4 & 25.4 & 41.7 & 83.3 & 25.0 & 58.3 & 50.0 \\
\hline
\end{tabular}

Note: OR—objective response, OS—overall survival, PFS—progression-free survival, SD—stable disease.

nosed AA have not been presented in peer-reviewed literature, but there were numerous studies on chemotherapy with TMZ and other agents and RT, and TMZ followed by RT.

Ultimately, almost all patients with AA treated with surgery, chemotherapy and radiation would relapse and die from PD. During the last eight years, there were nine Phase II clinical studies conducted for patients with recurrent AA, and the results were reported in peer-reviewed journals in English. There were only two Phase II trials exclusively designed for AA patients and neither proved efficacy [31] [32]. The results of three of these studies are shown in Table 4, and compared with the BT-09 study. The data indicate that in the BT-09 study there is a higher CR rate, but the patient population was smaller than in the other studies and included both newly-diagnosed and recurrent cases.

The responses observed in other high-grade brain tumors are interesting, but the population of patients is too small to draw conclusions. The additional studies provided data on the efficacy of ANP in GBM, PNET and BSG [9]-[17] [37].

An additional Phase II study may need to be designed to evaluate the efficacy of ANP in the treatment of LGG in adult patients. Two studies in LGA and optic pathway gliomas in children have been completed, and the results are being prepared for publication. The treatment with ANP is associated with a low percentage of serious ADEs (grade 3 and grade 4). Table 3 shows the incidence of ADEs in comparison with the other studies. There were no fatal ADEs, and the events were fully reversible. There was no chronic toxicity; and there was very good quality of survival.

The aim of this study was to provide preliminary information on the efficacy of the treatment with antineoplastons in patients diagnosed with inoperable primary brain tumors. The largest group of patients in the study consisted of the subjects diagnosed with AG and $86 \%$ of them diagnosed with AA. The authors believe that the responses determined in this group of patients are the most interesting and require further evaluation in additional clinical trials designed for the treatment of patients with AA. The limitation of the study is a diverse group of patients and small populations in the subgroups. The additional studies for newly-diagnosed AA and recurrent AA will provide more data on safety and efficacy. Such additional studies have been completed already and the results are published [14] [15]. A small group of seven patients diagnosed with low-grade tumors provided a high rate of disease control. Further studies will add data specific for each brain tumor, and continue to evaluate the long-term survival and quality of survival of this therapy.

\section{Acknowledgements}

The authors express their appreciation to the additional physicians involved in the care of the patients: Drs. Robert I. Lewy, Alejandro Marquis, Robert A. Weaver, Eva Kubove, Barbara Szymkowski, and Mohammad Khan. Preparation of the manuscript was provided by Carolyn Powers, Jennifer Pineda and Adam Golunski.

\section{References}

[1] Burzynski, S.R. (2004) The Present State of Antineoplaston Research. Integrative Cancer Therapies, 3, 47-58. 
http://dx.doi.org/10.1177/1534735403261964

[2] Burzynski, S.R., Kubove, E. and Burzynski, B. (1992) Phase II Clinical Trials of Antineoplastons A10 and AS2-1 Infusions in Astrocytoma. In: Adam, D., Ed., Recent Advances in Chemotherapy, Futuramed Publishers, Munich, 2506-2507.

[3] Burzynski, S.R., Janicki, T.J., Burzynski, G.S. and Marszalek, A. (2014) A Phase II Study of Antineoplastons A10 and AS2-1 in Children with High-Grade Glioma. Final Report (Protocol BT-06), and Review of Recent Trials. Journal of Cancer Therapy, 5, 565-577. http://dx.doi.org/10.4236/jct.2014.56065

[4] Burzynski, S.R., Lewy, R.I., Weaver, R., Janicki, T., Jurida, G., Khan, M., et al. (2004) Long-Term Survival and Complete Response of a Patient with Recurrent Diffuse Intrinsic Brain Stem Glioblastoma Multiforme. Integrative Cancer Therapies, 3, 257-261. http://dx.doi.org/10.1177/1534735404267748

[5] Burzynski, S.R., Weaver, R.A., Janicki, T., Szymkowski, B., Jurida, G., et al. (2005) Long-Term Survival of High-Risk Pediatric Patients with Primitive Neuroectodermal Tumors Treated with Antineoplastons A10 AS2-1. Integrative Cancer Therapies, 4, 168-177. http://dx.doi.org/10.1177/1534735405276835

[6] Burzynski, S.R., Janicki, T.J., Weaver, R.A. and Burzynski, B. (2006) Targeted Therapy with Antineoplastons A10 and AS2-1 of High-Grade, Recurrent, and Progressive Brainstem Glioma. Integrative Cancer Therapies, 5, 40-47. http://dx.doi.org/10.1177/1534735405285380

[7] Burzynski, S.R. (2006) Treatments for Astrocytic Tumors in Children: Current and Emerging Strategies. Pediatric Drugs, 8, 167-178. http://dx.doi.org/10.2165/00148581-200608030-00003

[8] Burzynski, S.R. (2007) Recent Clinical Trials in Diffuse Intrinsic Brainstem Glioma. Cancer Therapy, 5, 379-390.

[9] Burzynski, S.R., Janicki, T.J., Burzynski, G.S. and Marszalek, A. (2014) The Response and Survival of Children with Recurrent Diffuse Intrinsic Pontine Glioma Based on Phase II Study of Antineoplastons A10 and AS2-1 in Patients with Brainstem Glioma. Child's Nervous System, 30, 2051-2056. http://dx.doi.org/10.1007/s00381-014-2401-z

[10] Burzynski, S.R., Janicki, T.J., Burzynski, G.S. and Marszalek, A. (2014) Long-Term Survival (>13 Years) in a Child with Recurrent Diffuse Intrinsic Pontine Glioma: A Case Report. Journal of Pediatric Hematology/Oncology, 36, e433-e439. http://dx.doi.org/10.1097/MPH.0000000000000020

[11] Burzynski, S.R., Janicki, T.J. and Burzynski, G.S. (2014) A Phase II Study of Antineoplastons A10 and AS2-1 in Adult Patients with Recurrent Glioblastoma Multiforme. Final Report (Protocol BT-21). Journal of Cancer Therapy, 5, 946-956. http://dx.doi.org/10.4236/jct.2014.510100

[12] Burzynski, S.R., Janicki, T.J., Burzynski, G.S., Marszalek, A. and Brookman, S. (2014) A Phase II Study of Antineoplastons A10 and AS2-1 in Children with Recurrent, Refractory or Progressive Primary Brain Tumors. Final Report (Protocol BT-22). Journal of Cancer Therapy, 5, 977-988. http://dx.doi.org/10.4236/jct.2014.510102

[13] Burzynski, S.R., Janicki, T.J., Burzynski, G.S. and Marszalek, A. (2015) A Phase II Study of Antineoplastons A10 and AS2-1 in Patients with Brainstem Gliomas. The Report on Non-Diffuse Intrinsic Pontine Glioma (Protocol BT-11). Journal of Cancer Therapy, 6, 334-344. http://dx.doi.org/10.4236/jct.2015.64036

[14] Burzynski, S.R., Janicki, T.J., Burzynski, G.S. and Marszalek, A. (2015) A Phase II Study of Antineoplastons A10 and AS2-1 in Adult Patients with Newly-Diagnosed Anaplastic Astrocytoma. Final Report (Protocol BT-08). Cancer and Clinical Oncology, 4, 28-38. http://dx.doi.org/10.5539/cco.v4n1p28

[15] Burzynski, S.R., Janicki, T.J. and Burzynski, G.S. (2015) A Phase II Study of Antineoplastons A10 and AS2-1 Injections in Adult Patients with Recurrent Anaplastic Astrocytoma-Final Report (Protocol BT-15). Cancer and Clinical Oncology, 4, 13-23.

[16] Burzynski, S.R., Burzynski, G.S., Janicki, T.J. and Marszalek, A. (2014-2015) Complete Response and Long-Term Survival (>20 Years) of a Child with Tectal Glioma: A Case Report. Pediatric Neurosurgery, 50, 99-103. http://dx.doi.org/10.1159/000369907

[17] Burzynski, S.R., Burzynski, G.S., Marszalek, A., Janicki, T.J. and Martinez-Canca, J.F. (2015) Long-Term Survival (over 20 Years), Complete Response and Normal Childhood Development in Medulloblastoma Treated with Antineoplastons A10 and AS2-1. Journal of Neurology \& Stroke, 2, Article ID: 00054.

[18] Ogata, Y., Matono, K., Tsuda, H., Ushijima, M., Uchida, S., et al. (2015) Randomized Phase II Study of 5-Fluorouracil Hepatic Arterial Infusion with or without Antineoplastons as an Adjuvant Therapy after Hepatectomy for Liver Metastases from Colorectal Cancer. PLoS ONE, 10, e0120064. http://dx.doi.org/10.1371/journal.pone.0120064

[19] Ostrom, Q.T., Gittleman, H., Farah, P., Ondracek, A., Chen, Y., et al. (2013) CBTRUS Statistical Report: Primary Brain and Central Nervous System Tumors Diagnosed in the United States in 2006-2010. Neuro-Oncology, 15, ii1ii56. http://dx.doi.org/10.1093/neuonc/not151

[20] Louis, D.N., Ohgaki, H., Wiestler, O.D., Cavenee, W.K., Burger, P.C., et al. (2007) The 2007 WHO Classification of Tumours of the Central Nervous System. Acta Neuropathologica, 114, 97-109. 
http://dx.doi.org/10.1007/s00401-007-0243-4

[21] Dinnes, J., Cave, C., Huang, S. and Milne, R. (2002) A Rapid and Systematic Review of the Effectiveness of Temozolomide for the Treatment of Recurrent Malignant Glioma. British Journal of Cancer, 86, 501-505. http://dx.doi.org/10.1038/sj.bjc.6600135

[22] Yung, W.K., Prados, M.D., Yaya-Tur, R., Rosenfeld, S.S., Brada, M., et al. (1999) Multicenter Phase II Trial of Temozolomide in Patients with Anaplastic Astrocytoma or Anaplastic Oligoastrocytoma at First Relapse. Temodal Brain Tumor Group. Journal of Clinical Oncology, 17, 2762-2771.

[23] Scoccianti, S., Magrini, S.M., Ricardi, U., Detti, B., Krengli, M., et al. (2012) Radiotherapy and Temozolomide in Anaplastic Astrocytoma: A Retrospective Multicenter Study by the Central Nervous System Study Group of AIRO (Italian Association of Radiation Oncology). Neuro-Oncology, 14, 798-807. http://dx.doi.org/10.1093/neuonc/nos081

[24] Wick, W., Hartmann, C., Engel, C., Stoffels, M., Felsberg, J., et al. (2009) NOA-04 Randomized Phase III Trial of Sequential Radiochemotherapy of Anaplastic Glioma with Procarbazine, Lomustine, and Vincristine or Temozolomide. Journal of Clinical Oncology, 27, 5874-5880. http://dx.doi.org/10.1200/JCO.2009.23.6497

[25] Sarganas, G., Orzechowski, H.D., Klimpel, A., Thomae, M., Kauffmann, W., et al. (2012) Severe Sustained Cholestatic Hepatitis Following Temozolomide in a Patient with Glioblastoma Multiforme: Case Study and Review of Data from the FDA Adverse Event Reporting System. Neuro-Oncology, 14, 541-546. http://dx.doi.org/10.1093/neuonc/nos056

[26] Grossman, S.A., Ye, X., Lesser, G., Sloan, A., Carraway, H., et al. (2011) Immunosuppression in Patients with HighGrade Gliomas Treated with Radiation and Temozolomide. Clinical Cancer Research, 17, 5473-5480. http://dx.doi.org/10.1158/1078-0432.CCR-11-0774

[27] Chang, S.M., Kuhn, J.G., Robins, H.I., Schold, S.C., Spence, A.M., et al. (1999) Phase II Study of Phenylacetate in Patients with Recurrent Malignant Glioma: A North American Brain Tumor Consortium Report. Journal of Clinical Oncology, 17, 984-990.

[28] Wen, P.Y., Macdonald, D.R., Reardon, D.A., Cloughesy, T.F., Sorensen, A.G., et al. (2010) Updated Response Assessment Criteria for High-Grade Gliomas: Response Assessment in Neuro-Oncology Working Group. Journal of Clinical Oncology, 28, 1963-1972. http://dx.doi.org/10.1200/JCO.2009.26.3541

[29] Weller, M., Cloughesy, T., Perry, J.R. and Wick, W. (2013) Standards of Care for Treatment of Recurrent Glioblastoma-Are We There Yet? Neuro-Oncology, 15, 4-27. http://dx.doi.org/10.1093/neuonc/nos273

[30] MedCalc Software bvba, Ostend, Belgium. http://www.medcalc.org

[31] Chamberlain, M.C., Tsao-Wei, D.D. and Groshen, S. (2006) Salvage Chemotherapy with Cyclophosphamide for Recurrent Temozolomide-Refractory Anaplastic Astrocytoma. Cancer, 106, 172-179. http://dx.doi.org/10.1002/cncr.21582

[32] Chamberlain, M.C., Wei-Tsao, D.D., Blumenthal, D.T. and Glantz, M.J. (2008) Salvage Chemotherapy with CPT-11 for Recurrent Temozolomide-Refractory Anaplastic Astrocytoma. Cancer, 112, 2038-2045. http://dx.doi.org/10.1002/cncr.23404

[33] Fine, H.A., Dear, K.B., Loeffler, J.S., Black, P.M. and Canellos, G.P. (1993) Meta-Analysis of Radiation Therapy with and without Adjuvant Chemotherapy for Malignant Gliomas in Adults. Cancer, 71, 2585-2597. http://dx.doi.org/10.1002/1097-0142(19930415)71:8<2585::AID-CNCR2820710825>3.0.CO;2-S

[34] Stewart, L.A. (2002) Chemotherapy in Adult High-Grade Glioma: A Systematic Review and Meta-Analysis of Individual Patient Data from 12 Randomised Trials. The Lancet, 359, 1011-1018. http://dx.doi.org/10.1016/S0140-6736(02)08091-1

[35] Stupp, R., Reni, M., Gatta, G., Mazza, E. and Vecht, C. (2007) Anaplastic Astrocytoma in Adults. Critical Reviews in Oncology/Hematology, 63, 72-80. http://dx.doi.org/10.1016/j.critrevonc.2007.03.003

[36] Glantz, M., Chamberlain, M., Liu, Q., Litofsky, N.S. and Recht, L.D. (2003) Temozolomide as an Alternative to Irradiation for Elderly Patients with Newly Diagnosed Malignant Gliomas. Cancer, 97, 2262-2266. http://dx.doi.org/10.1002/cncr.11323

[37] Burzynski, S.R., Burzynski, G.S. and Janicki, T.J. (2014) Recurrent Glioblastoma Multiforme. A Strategy for Long-Term Survival. Journal of Cancer Therapy, 5, 957-976. http://dx.doi.org/10.4236/jct.2014.510101 